\title{
Diagnostic Value of Procalcitonin and Interleukin-6 on Early Postoperative Pneumonia after Adult Cardiac Surgery: A Prospective Observational Study
}

\author{
Man Xie, ${ }^{1}$ Ya Ting Chen, ${ }^{1}$ He Zhang, ${ }^{1}$ Hai Tao Zhang, ${ }^{1}$ Ke Pan, ${ }^{2}$ Xiao Fang Chen, ${ }^{1}$ Xiao Li Xie, ${ }^{1}$ \\ ${ }^{1}$ Department of Cardio-Thoracic Surgery, Nanjing Drum Tower Hospital, The Affiliated Hospital of Nanjing University Medical \\ School, Nanjing, Jiangsu, China; ${ }^{2}$ Nanjing Drum Tower Hospital, The Affiliated Clinical College of Xuzhou Medical University
}

\section{ABSTRACT}

Background: Postoperative pneumonia (PP) is the most common primary infection after cardiac surgery, increasing the hospitalization expense and causing the consumption of healthcare resources. This study aimed to investigate the diagnostic value of procalcitonin (PCT) and interleukin-6 (IL-6) on early postoperative pneumonia after adult cardiac surgery.

Methods: In this prospective observational study, patients with pneumonia and age- and sex-matched cases in our center from October 10, 2020 to January 31, 2021 were included. Patients diagnosed with pneumonia in this study needed meet both clinical and microbiological diagnostic criteria. Blood samples were collected in all patients from postoperative day (POD) 1 to postoperative day 5 to detect PCT, IL-6, white blood cell count, and C-reactive protein. The diagnostic performance of different biomarkers was evaluated by the receiver operating characteristic curves and the area under the curves.

Results: Our study enrolled 272 patients, including 24 patients with postoperative pneumonia and 248 age- and sexmatched cases. From POD1 to POD5, the absolute value of PCT and PCT variations showed diagnostic significance for pneumonia $(P<.05)$; the diagnostic value of the absolute value of IL-6 and IL-6 variations was not satisfying. White blood cell count showed no differences; C-reactive protein had no diagnostic value before POD4. Multivariable logistic regression showed that PCT variation and IL-6 variation from POD 3 to POD1 were the strongest risk factors for postoperative pneumonia [OR:12.50, 95\% CI: (3.40-45.5); OR:13.71, 95\% CI: (1.11-168.47)]. According to the above results, we defined the PL Index. PL Index showed the best diagnostic value among those biomarkers in POD3 [AUC: 0.90, 95\% CI: (0.79-0.95)]. Multivariable logistic regression showed PL Index POD3 has significant correlation with postoperative pneumonia [OR:1.23, 95\% CI: (1.11-1.37), $P=.041]$.

Conclusions: PCT variation and IL-6 were more accurate than C-reactive protein and white blood cell count to predict early postoperative pneumonia, but the diagnostic properties

Received September 12, 2021; accepted October 8, 2021.

Correspondence: Xiao Li Xie and Xiao Fang Chen, Department of CardioThoracic Surgery, Nanjing Drum Tower Hospital, The Affiliated Hospital of Nanjing University Medical School, Number 321 Zhongshan Road, Nanjing, fiangsu, 210008,China; (e-mails: xiaolixieglyy@163.com and cxfzyp@yeah.net). of PCT could not be observed during the first three postoperative days due to the inflammatory process. By combining the variations of PCT and IL-6, we defined the PL Index, which shows the best diagnostic ability on early postoperative pneumonia after adult cardiac surgery.

\section{INTRODUCTION}

Postoperative pneumonia $(\mathrm{PP})$ is the most common infection after cardiac surgery, inducing 8.9 times higher risk of mortality and directly prolonging hospitalization time [Ailawadi 2017]. PP also increases hospitalization costs, causing the consumption of healthcare resources [He 2014; Hulzebos 2006; Kinlin 2010]. Therefore, diagnosing PP earlier may be beneficial to improve patient prognosis and save on medical expenses.

In the early days after cardiac surgery with cardiopulmonary bypass $(\mathrm{CPB})$, systemic inflammatory response syndrome (SIRS) can elevate white blood cell count (WBC), C-reactive protein (CRP) and cover infectious symptoms, misleading the diagnosis of PP [Wan 1997]. Meanwhile, the traditional gold standard for PP diagnosis - microbiological cultures may have negative results or take days to yield cultures with identifiable quantities, which makes it difficult to be used for early diagnosis of pneumonia [Chanderraj 2018]. Therefore, detecting specific biomarkers to diagnose $\mathrm{PP}$ earlier would be beneficial to come up with a fast therapeutic strategy.

Currently, several biologic indicators have been regarded as helpful markers for early diagnosis of infections in clinical practices, most common among them are procalcitonin (PCT) and interleukin-6 (IL-6). As an acute-phase protein, serum PCT levels increase significantly in infection and sepsis [Becker 2008]. It's considered a helpful biomarker for diagnosis of infections [van Rossum 2004; Wacker 2013]. $\mathrm{Li}$ et al reported that monitoring PCT would be beneficial to differentiate infection after pediatric cardiac surgery [Li 2017]. Another potential biomarker may be IL-6. It's a multifunctional cytokine that plays an important role in the acute-phase reaction after inflammation and infections [Tanaka 2014]. Previous studies showed that IL-6 had good early diagnostic value in patients with infection and sepsis [Steinmetz 1995; Le Moine 1994]. Therefore, both PCT and IL-6 may help with early diagnosis of infections. However, it still is unknown the diagnostic value of PCT and IL-6 on early PP after adult cardiac surgery. 
Table 1. Clinical Baseline Data of Patients in the Non-PP and PP Groups

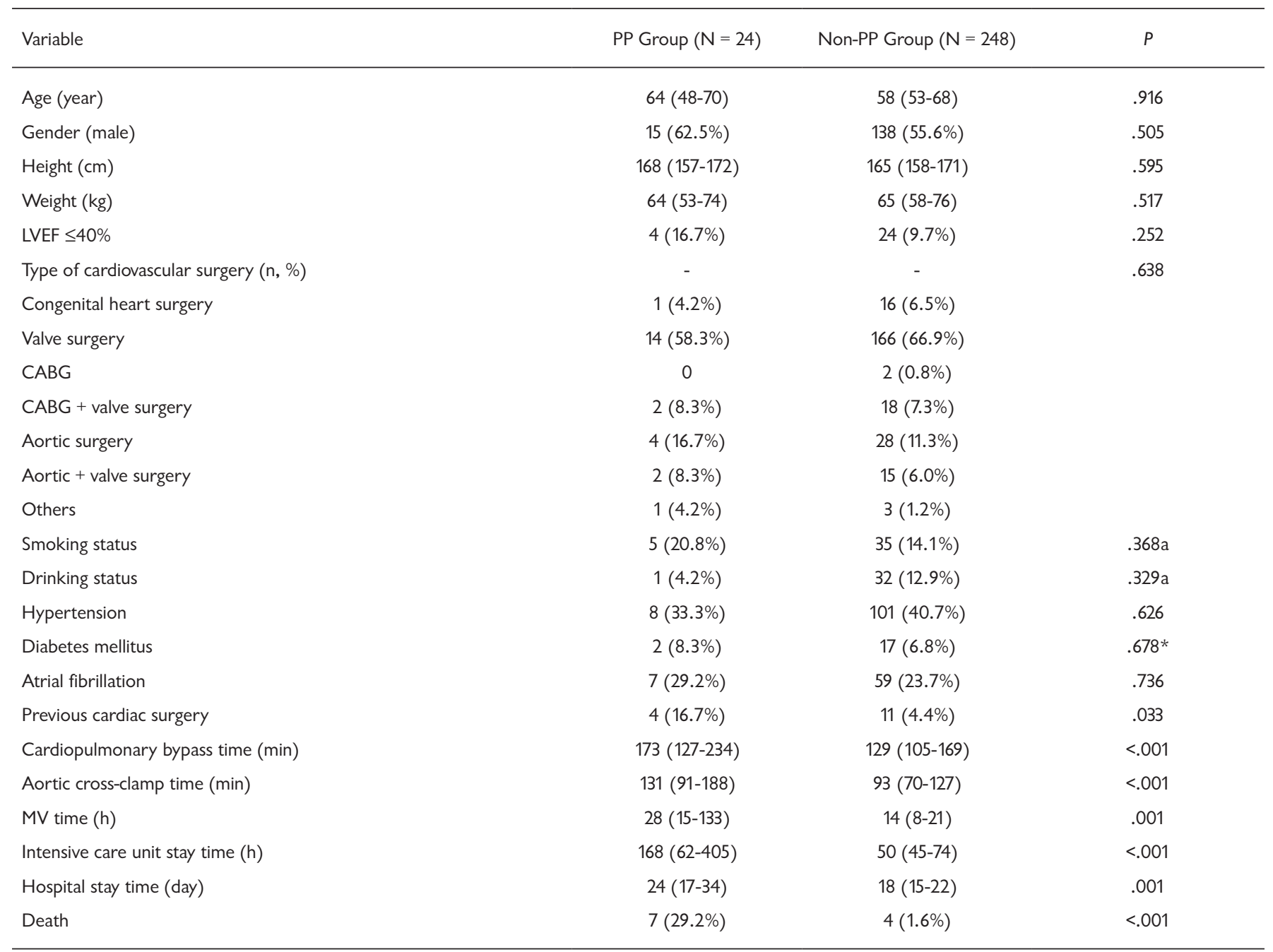

*Fisher exact test; CABG, coronary artery bypass grafting; PP, postoperative pneumonia; Median (interquartile range); MV, mechanical ventilation; LVEF, left ventricular ejection fraction

To our knowledge, there is no study exploring whether these markers could diagnose PP in the first few days after adult cardiac surgery. Therefore, the primary aim of our study was to assess the diagnostic value of PCT and IL-6 on early PP after adult cardiac surgery.

\section{MATERIALS AND METHODS}

\section{Study design and patient characteristics}

This work was a single-center, prospective and observational study. The trial was registered at Chinese Clinical Trial Register (www.chictr.org.cn, ChiCTR2000038762). The study was conducted at Cardiothoracic Surgery of Nanjing Drum Tower Hospital and approved by the Medical Ethics Committee of Affiliated Nanjing Drum Tower Hospital, Nanjing University Medical College
(2020-249-01). All patients gave informed consent. Inclusion and exclusion criteria are as follows: 1) Adult patients (between 18 and 80 years old) undergoing cardiovascular surgery with $\mathrm{CPB}$, and 2) Written informed consent to participate in the study. Exclusion criteria: 1) Preoperative body temperature $\geq 38^{\circ}$ or white blood cell count $>10000$ cells/ $\mathrm{mm} 3$ or serum procalcitonin $>0.5 \mathrm{ng} / \mathrm{ml}$; 2) Patients undergoing cardiovascular surgery for trauma, infective endocarditis, neoplasms and malignant tumors; 3) Patients who were diagnosed with any other infectious diseases (pneumonia, sepsis, etc.); 4) Patients who were diagnosed with inflammatory immune diseases or connective tissue diseases; 5) Pregnant or lactating women; 6) Patients with missing clinical data due to perioperative death or other reasons.

We continuously included patients with PP and matched the controls at a ratio of 1:10 with age \pm 3 years and sex in our center from October 10, 2020 to January 31, 2021. There 


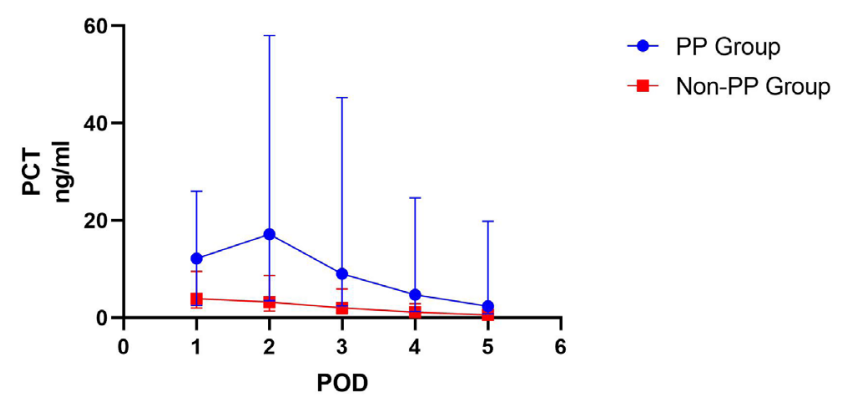

Figure 1. PCT levels from POD1 to POD5

were 272 patients who met the eligibility criteria, including 24 consecutive postoperative pneumonia patients (PP group) and 248 age- and sex-matched cases (non-PP group).

\section{$C P B$ and perioperative management}

All operations were performed via median sternotomy and CPB. The ascending aorta was cannulated with a patient size-appropriate cannula. Venous cannulations were chosen with separate cannulas in the superior and inferior vena cava. The initial volume of the antegrade cold blood cardioplegia solution (4:1 cardioplegia solution to blood ratio) was twice the volume needed for the cessation of all cardiac electrical activity but never less than $1,000 \mathrm{ml}$. Cardiac arrest was maintained by the retrograde infusion of $300 \mathrm{ml}$ of blood cardioplegia solution (8:1 cardioplegia solution to blood ratio) every 20 minutes. Occasionally, intermittent antegrade cold blood cardioplegia was used, according to the surgeon's preferences. Prophylactic antibiotic therapy routinely was given in the perioperative period to prevent infections.

The first dose of antibiotic was injected intravenously within 60 minutes of the incision. Successive dosages should be administered every 3-4 h during surgery [Engelman 2007]. In addition, we continued to administer adequate antibiotics in the first 48 hours after the operation, as recommended by the guidelines. We routinely chose the first-generation cephalosporins as prophylactic intravenous antibiotics and may be use vancomycin, when clinicians feel it is necessary [Engelman 2007; Edwards 2006; Vos 2018].

\section{Biologic measurement}

Blood samples for PCT, IL-6, CRP, and WBC measurement were collected separately before surgery and daily until the fifth postoperative day. PCT was measured by an immune luminometric assay whose detection limit range from 0.05 to $25 \mathrm{ng} / \mathrm{ml}$ and normal value was less than $0.5 \mathrm{ng} / \mathrm{ml}$. CRP was measured by automatic laser nephelometry with a normal value less than $8 \mathrm{mg} / \mathrm{L}$. WBC was determined by using an automatic counter with a normal value between 4,000 and 10,000 cells $/ \mathrm{mm} 3$. The coefficient of variation of the measurements was less than $5 \%$. All the patients underwent sputum culture using alveolar lavage with fiberoptic bronchoscopy in POD1-3. Pleural effusion culture examination also used to detect pathogenic bacteria.

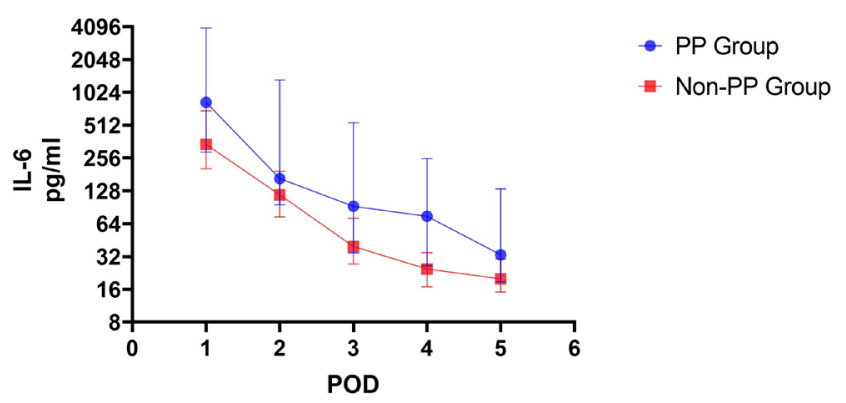

Figure 2. IL-6 levels from POD1 to POD5

\section{Diagnosis of postoperative pneumonia}

Sputum culture was performed at least twice with fiberoptic bronchoscope or alveolar lavage fluid. Pathogenic bacteria were detected in sputum culture, and the first positive result appeared within 48 hours after surgery. The PP was defined when pathogenic bacteria were detected at least twice and new detection of lung infiltrates on chest radiograph associated with at least two of three clinical features: 1) fever more than $38^{\circ} \mathrm{C}$; 2) leukocytosis or leukopenia; 3) purulent secretion [Guidelines 2005; Kalil 2016].

The final diagnosis of PP was determined by two independent experts in regard to the complete clinical records. In cases of disagreement between the two clinical experts, a third expert reached a consensus. Each final diagnosis was classified as microbiologically confirmed, probable, possible, or absent [Calandra 2005]. The final diagnosis was reached when it was classified as microbiologically confirmed by the experts and ruled out when the experts classified it as absent, probable, or possible. Experts were blinded for PCT, CRP, IL-6, and WBC. Finally, patients were divided into two groups: the non-PP group, who did not develop any postoperative infection within 5 days after surgery, and the PP group, who developed pneumonia within 2 days after surgery.

\section{Data collection}

Data were recorded prospectively. Data collected included demographic characteristics and preoperative diagnosis. Intraoperative data recorded were surgical methods, extracorporeal circulation time, and ascending aorta occlusion time. Mechanical ventilation time, intensive care unit time, hospital stay, imaging examination results, and microbiological cultures results also were recorded through the electronic case system. Serum concentration of PCT, CRP, and IL-6 were recorded during POD1-5. Variations of PCT were calculated by the equation: (PCTPODn-PCTPOD1)/PCT POD1. PCTPODn was PCT serum concentration at PODn; IL-6 variation was defined same as the method mentioned above.

\section{Sample size}

In this study, we evaluated the diagnostic value of biomarkers based on the receiver operating characteristics (ROC) curves and area under the curve (AUC). Therefore, we calculated the sample size required for the study based on AUC. Power Analysis and Sample Size (version 15.0) was used for 
Table 2. PCT, IL-6 Serum Concentrations and Variation of Patients in the PP and Non-PP Groups

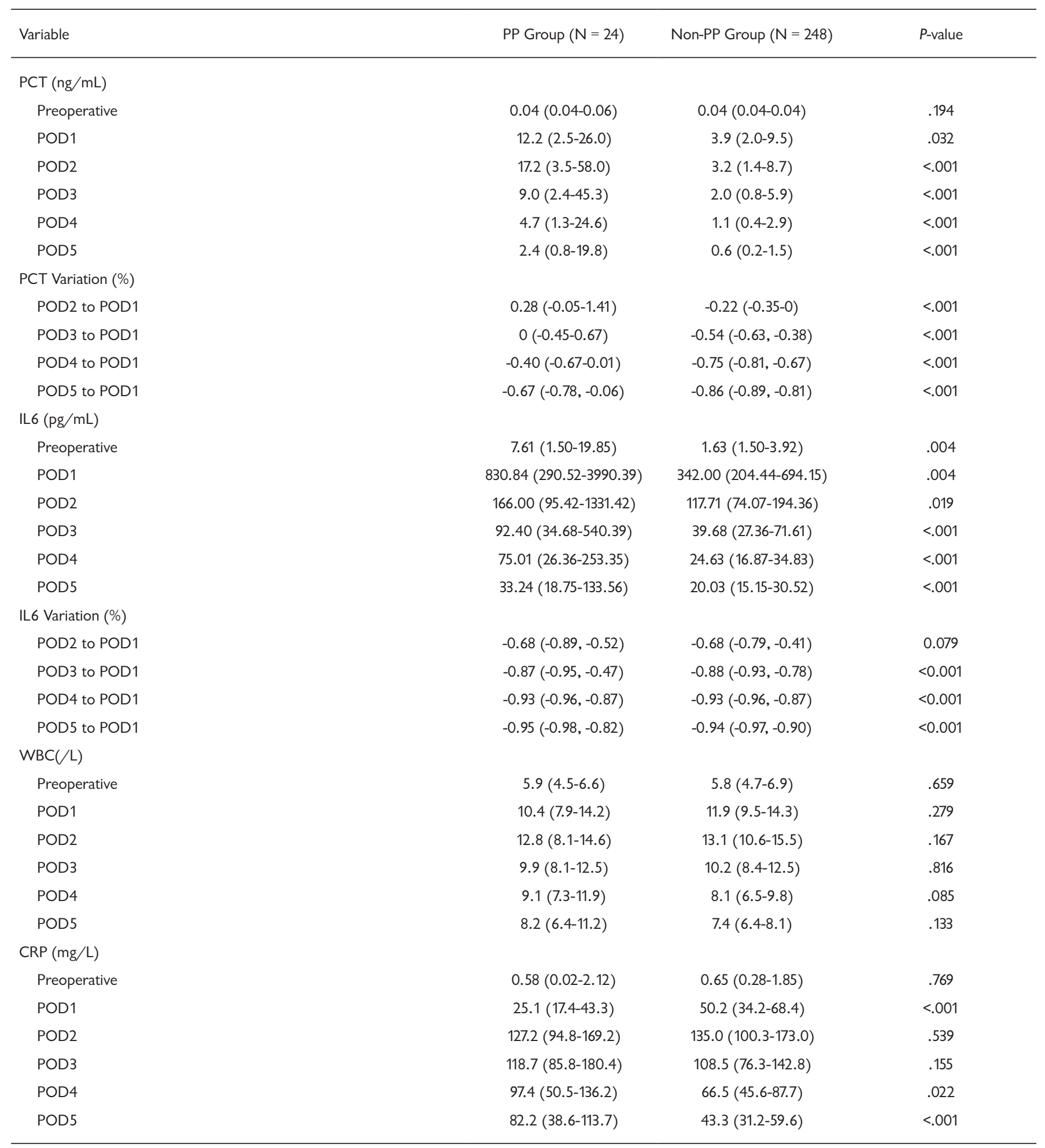

Median (interquartile range); POD, postoperative day 


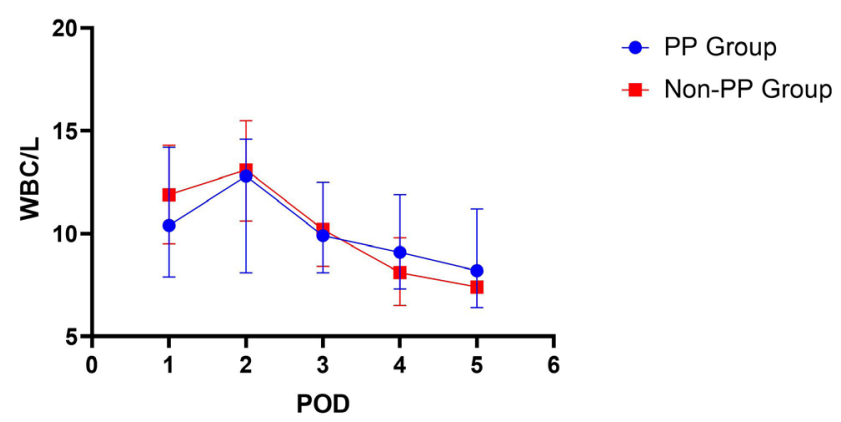

Figure 3. WBC from POD1 to POD5

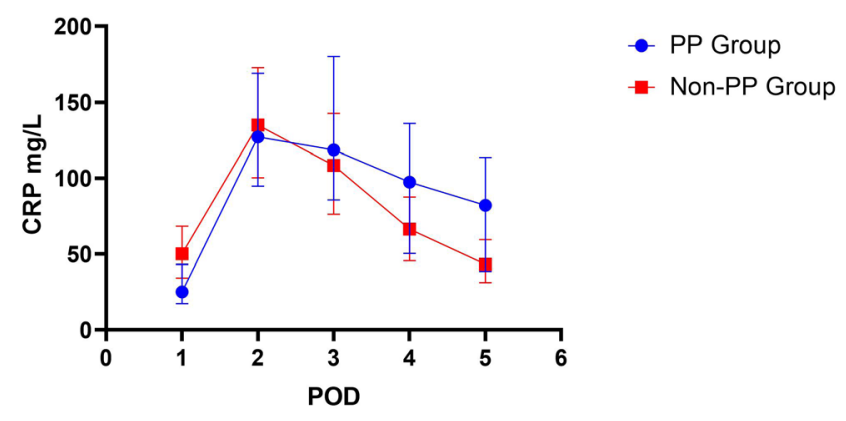

Figure 4. CRP levels from POD1 to POD5

calculating the required sample size. In previous study, the AUC of procalcitonin to diagnose infection ranged 0.81 0.90 [Li 2017; Klingele 2016]. Therefore, we set the AUC of this study to 0.85 . Considering a two-sided significance level of $5 \%$ $(\alpha)$ and a test power of $80 \%(1-\beta), 24$ patients with postoperative pneumonia would be required. Because of the 1:10 ageand sex-match, our control group needs at least 240 patients.

\section{Statistical analysis}

Statistical analyses were performed by SPSS, version 26.0 (SPSS, Chicago, IL). Normally distributed variables were presented as mean \pm standard deviation and compared by Student's t-test. Nonparametric continuous variables were presented as median and interquartile range (IQR), compared by the Mann-Whitney $\mathrm{U}$ test. Continuous variables were determined to be normal in distribution by the Shapiro-Wilk test. Categorical variables were presented as absolute numbers and percentages, equated by the chi-square test or Fisher's exact test.

The diagnostic performance of biomarkers was demonstrated with ROC analysis and AUC. The Youden index with highest sum of sensitivity and specificity was used to determine the optimal cutoff values [Youden 1950]. Results were expressed for AUC as mean and 95\% confidence interval (CI). For additional analyses, covariates in parametric and non-parametric test, which have clinical importance and significant differences, were entered into a multivariable logistic regression model designed to assess the independent

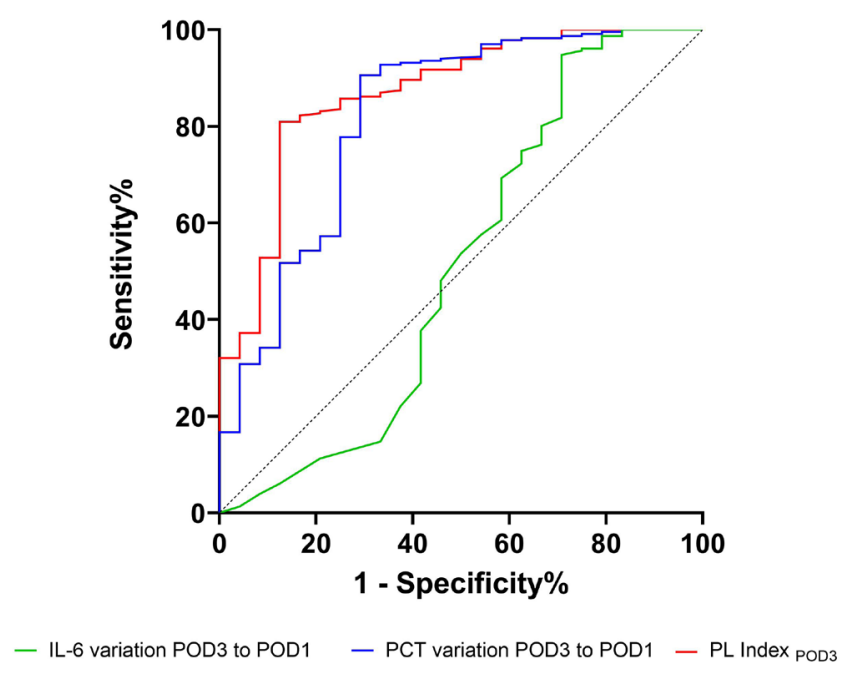

Figure 5. Comparison of the receiver operating characteristic curves showing the predictive value of PCT variation (from POD3 to POD1), IL-6 variation (from POD3 to POD1), and PL Index (POD3) for the diagnosis of postoperative pneumonia.

associated factors with PP. Collinearity diagnostics were performed using tolerance estimates for individual variables in a linear regression model. The two groups were analyzed using repeated measures analysis of variance (ANOVA). For all statistical tests, a $P$ value $<.05$ was considered significant.

\section{RESULTS}

\section{Patient characteristics}

During the study period, 398 patients were admitted to the CICU after cardiac surgery. There were 272 patients included in this study. Of these patients, 153 (56.3\%) were male and $119(43.7 \%)$ female, with a 48-70 age range. Twenty-four patients $(8.8 \%)$ were categorized into the PP group, and 248 patients $(91.2 \%)$ without infections were categorized into the non-PP group.

Characteristics of the patients in the two groups were presented in Table 1. No significant differences were found in age, sex, cardiac diagnosis, and preoperative complications. The PP group presented longer CPB time ( $\mathrm{min}$ ) and aortic cross-clamp time (min) compared with the non-PP group. The PP group had much higher mortality than the non-PP group $(24 \%$ versus $1.6 \%, P<.001)$. Patients in the PP group had prolonged mechanical ventilation (median: $28 \mathrm{~h}$, IQR: [15-133] h versus median: 14 , IQR: [2-21] h, $P<.001)$, intensive care unit stay time (median: $168 \mathrm{~h}, \mathrm{IQR}$ : [62-405] h versus median: $50 \mathrm{~h}$, IQR: [45-74] h, $P<.001)$, and hospital stay time (median: $24 \mathrm{~h}, \mathrm{IQR}$ : [17-34] h versus median: $18 \mathrm{~h}$, IQR: [15-22] h, $P=.001)$ compared with the non-PP group.

\section{Biomarkers in study groups}

PCT, IL-6, WBC, and CRP were measured and analyzed in all enrolled patients excluded with $\mathrm{WBC} \geq 10 \times 10^{9} / \mathrm{L}$ or 
Table 3. Comparison of AUC and Efficiency of WBC- CRP- PCT and PCT Variation in the Diagnosis of Postoperative Infection

\begin{tabular}{|c|c|c|c|c|}
\hline \multicolumn{5}{|l|}{ WBC $\left(10^{9} / \mathrm{L}\right)$} \\
\hline POD2 & $0.394(0.264-0.524)$ & 9.6 & 0.609 & 0.124 \\
\hline POD3 & $0.474(0.342-0.606)$ & 15.4 & 0.174 & 0.931 \\
\hline POD5 & $0.589(0.446-0.731)$ & 8.45 & 0.522 & 0.720 \\
\hline \multicolumn{5}{|l|}{$\mathrm{CRP}(\mathrm{mg} / \mathrm{L})$} \\
\hline POD1 & $0.266(0.144-0.387)$ & 28.3 & 0.391 & 0.165 \\
\hline POD2 & $0.446(0.317-0.575)$ & 136.7 & 0.304 & 0.486 \\
\hline POD3 & $0.579(0.446-0.731)$ & 81.5 & 0.870 & 0.307 \\
\hline POD1 & $0.63(0.50-0.76)$ & 15.6 & 0.50 & 0.83 \\
\hline POD2 & $0.74(0.63-0.85)$ & 8.6 & 0.67 & 0.75 \\
\hline POD3 & $0.75(0.65-0.85)$ & 7.7 & 0.58 & 0.82 \\
\hline POD4 & $0.76(0.66-0.86)$ & 2.7 & 0.67 & 0.75 \\
\hline POD5 & $0.77(0.67-0.87)$ & 1.7 & 0.67 & 0.78 \\
\hline \multicolumn{5}{|l|}{ PCT variation } \\
\hline POD2 to POD1 & $0.81(0.70-0.91)$ & -0.08 & 0.17 & 0.67 \\
\hline POD3 to POD1 & $0.82(0.71-0.92)$ & -0.15 & 0.71 & 0.90 \\
\hline POD4 to POD1 & $0.83(0.73-0.93)$ & -0.57 & 0.75 & 0.89 \\
\hline \multicolumn{5}{|l|}{ IL6 (pg/ml) } \\
\hline POD4 & $0.80(0.67-0.91)$ & 43.83 & 0.67 & 0.85 \\
\hline POD5 & $0.72(0.60-0.85)$ & 104.30 & 0.46 & 0.98 \\
\hline \multicolumn{5}{|l|}{ IL6 variation } \\
\hline POD2 to POD1 & $0.44(0.30-0.58)$ & 1.02 & 0.83 & 0.99 \\
\hline POD3 to POD1 & $0.52(0.36-0.67)$ & -0.48 & 0.30 & 0.95 \\
\hline POD4 to POD1 & $0.53(0.38-0.69)$ & -0.69 & 0.25 & 0.96 \\
\hline POD5 to POD1 & $0.48(0.33-0.63)$ & -0.81 & 0.25 & 0.89 \\
\hline \multicolumn{5}{|l|}{ PL Index } \\
\hline PL Index POD2 & $0.74(0.62-0.86)$ & 1.28 & 0.50 & 0.44 \\
\hline PL Index POD3 & $0.90(0.79-0.95)$ & -14.77 & 0.88 & 0.68 \\
\hline PL Index POD4 & $0.88(0.80-0.95)$ & -20.38 & 0.87 & 0.65 \\
\hline PL Index POD5 & $0.82(0.72-0.91)$ & -22.30 & 0.79 & 0.55 \\
\hline
\end{tabular}

POD, postoperative day 
Table 4. Multivariate Analysis Between the PP and the Non-PP Groups

\begin{tabular}{lcc}
\hline Variable & OR $(95 \% \mathrm{Cl})$ & $P$ \\
\hline PCT Variation POD3 to POD1 & $12.50(3.40-45.5)$ & $<.001$ \\
IL6 Variation POD3 to POD1 & $13.71(1.11-168.47)$ & .041 \\
Previous cardiac surgery & $0.19(0.04-0.87)$ & .032 \\
MV time & $1.00(0.99-1.01)$ & .742 \\
Cardiopulmonary bypass time (min) & $1.01(1.00-1.01)$ & .016 \\
Hospital stay & $1.083(1.018,1.153)$ & .012
\end{tabular}

PCT $>0.5 \mathrm{ng} / \mathrm{ml}$ or IL-6 $>10 \mathrm{pg} / \mathrm{ml}$ before surgery. There were no significant differences in PCT (median: $0.04 \mathrm{ng} /$ ml, IQR: [0.04-0.06] ng/ml versus median: $0.04 \mathrm{ng} / \mathrm{ml}$, IQR: [0.04-0.04] ng/ml, $P=.139$ ), WBC (median: $5.9 \times 109 / \mathrm{L}$, IQR: [4.5-6.6] $\times 10^{9} / \mathrm{L}$ versus median: $5.8 \times 10^{9} / \mathrm{L}$, IQR: [4.76.9] $\times 109 / \mathrm{L}, P=.659$ ), and CRP (median: $0.58 \mathrm{mg} / \mathrm{L}, \mathrm{IQR}$ : [0.02-2.12] mg/L versus median: $0.65 \mathrm{mg} / \mathrm{L}, \mathrm{IQR}:$ [0.28-1.85] $\mathrm{mg} / \mathrm{L}, P=.769$ ) before surgery between the two groups. IL-6 in the PP group were higher than the non-PP group (median: $7.61 \mathrm{pg} / \mathrm{ml}$ IQR: [1.50-19.85] pg/ml versus median: $1.63 \mathrm{pg} /$ ml, IQR: [1.50-3.92] pg/ml, $P=.004)$.

In the PP group, PCT concentration increased in the first two days after surgery, peaked on POD2 and then began to decrease. In the non-PP group, PCT concentration peaked on POD1. During POD 1-5, the non-PP group had significant lower PCT concentration compared with the PP group $(P<$ $.01)$ (Figure 1). The value of PCT variation was significantly higher in the non-PP group than in the infected ones between POD3 and POD5; from the 95\% CI, we observed some positive values in the PP group, indicating PCT concentration increased in some infected patients, whereas in the non-PP group, all values of PCT variation were negative (Table 2).

IL-6 concentration peaked on POD1, and the PP group had higher IL-6 concentration compared with the non-PP group during POD $1-5(P<.05)$ (Figure 2$)$ The value of IL-6 variation was significantly higher in the non-PP group than in the PP group between POD3 and POD5 (Table 2).

WBC peaked on POD2 and had no difference between the two groups from POD1 to POD5 $(P<.01)$ (Figure 3$)$. There were no differences between the two groups $(P>$ $.05 / 5)$ after Bonferroni correction. CRP concentration of the two groups peaked on the POD2, respectively $(P<.01)$ (Figure 4). Then, the CRP concentration kept decreasing, but there were no differences between the two groups until POD4 (Table 2). After Bonferroni correction, CRP had differences between the two groups on POD1 and POD5 $(P<$ $.05 / 5)$. In the PP group, CRP concentration was lower on POD1 $(P<.01)$.

Diagnostic value of biomarkers and PCT, IL-6 variations: We calculated AUCs of all biomarkers and variations of PCT and IL-6. AUC of PCT on POD 2-5, IL-6 on POD 3-5, and PCT variation from POD1 to POD5 were $>0.7$ (Table 3). The PCT variations had best diagnostic value (AUC>0.8) (Figure 5). Detailed data are shown in Table 3.
Table 5. Multivariate Analysis Between the PP and the NonPP Groups

\begin{tabular}{lcc}
\hline Variable & OR $(95 \% \mathrm{Cl})$ & $P$ \\
\hline PL Index POD3 & $1.23(1.11-1.37)$ & .041 \\
PCT variation (POD3 to POD1) & $1.32(0.15-11.34)$ & .799 \\
Previous cardiac surgery & $5.54(1.25-24.55)$ & .032 \\
MV time (h) & $1.00(0.99-1.01)$ & .742 \\
Cardiopulmonary bypass time (min) & $1.01(1.00-1.02)$ & .016
\end{tabular}

Multivariate analysis between the PP and non-PP groups

Multivariable logistic regression found that PCT variation from POD3 to POD1, IL-6 variation from POD3 to POD1, previous cardiac surgery, $\mathrm{MV}$ time and cardiopulmonary bypass time were independently correlated to PP. Among them, PCT variation and IL-6 variation were the strongest risk factors for $\mathrm{PP}$ [OR: 12.50, 95\% CI: (3.40-45.5); OR: 13.71, 95\% CI: (1.11-168.47)] (Table 4).

\section{PL Index}

For earlier and more accurate diagnosis of postoperative pneumonia, we defined the PL Index based on the following formula, according to the multivariable logistic regression results:

$\mathrm{PL}$ Index PODn $=13{ }^{*} \mathrm{PCT}$ Variation $\mathrm{PODn}$ to POD $1+14 *$ IL-6 Variation PODn to POD1

Then, we calculated AUCs of PL Index on POD 2-5; all the ROC were $>0.7$ (Table 3). AUC of PL Index on POD3 was 0.90 (Figure 5). Multivariable logistic regression showed that PL Index on POD3, previous cardiac surgery and cardiopulmonary bypass time were independently correlated to PP. PL Index on POD3 had higher correlation compared with PCT variation from POD3 to POD1 [OR:1.23, 95\% CI: (1.11-1.37), $P=.041$ versus OR: $1.32,95 \% \mathrm{CI}:(0.15-11.34)$, $P=.799]$ (Table 5).

\section{DISCUSSION}

After cardiac surgery with cardiopulmonary bypass (CPB), systemic inflammatory response syndrome (SIRS) makes the early diagnosis of PP more difficult. Detecting specific biomarkers to diagnose the occurrence of PP earlier may be beneficial to come up with a fast and moderate therapeutic strategy, improve patient prognosis, and save on medical expenses. As potential biomarkers, the diagnosis value of procalcitonin (PCT) and interleukin-6(IL-6) on early PP remains controversial. Therefore, we presented a single-center, prospective and observational study to assess the diagnostic value of PCT and IL-6 on early PP after adult cardiac surgery. Our study demonstrated that (I) PCT absolute value and PCT variation both had good accuracy with identifying PP in the early days after cardiac surgery; (II) Diagnostic value of IL-6 absolute value and IL-6 variation were worse than PCT absolute value and PCT variation, but still superior to CRP and WBC; and 
(III) In order to diagnose PP earlier and more accurately, we built a PL Index through the combination of PCT variation with IL-6 variation, which has better diagnostic value on PP than each alone.

Several studies have reported that the PCT concentration increases rapidly after microbial invasion and is correlated with the severity of infection [Assicot 1993; Dandona 1994]. Based on these characteristics, PCT is considered a helpful biomarker for diagnosis of bacterial infections [Wacker 2013]. After cardiac surgery, higher PCT levels were observed in infected patients compared with non-infected patients, which could help predict postoperative complications [Macrina 2005; Baykut 2000; Kin 2003; Dörge 2003]. Despite being affected by SIRS after cardiac surgery, PCT still has good diagnostic value of bacterial infections after cardiac surgery, and CRP and WBC had not [Jebali 2007]. In pediatric patients with complex congenital heart disease, PCT was more accurate than CRP and WBC to predict early postoperative infection; monitoring PCT would be helpful to differentiate infection after pediatric cardiac surgery [Wacker 2013]. We described the postoperative PCT serum concentrations and variations after cardiac surgery with CPB (Table 2 and Figure 1), which was in agreement with previous studies. Our study observed an elevated PCT concentration within the first 2 days after cardiac surgery in the PP group, while in non-infected patients, the PCT concentration had only transient rise on POD1, then progressively decreased. Besides absolute value, the PCT variations in the PP group were less than the non-PP group significantly since POD2. The characteristics above indicated that PCT was a better predictor of postoperative pneumonia during POD1 to POD5, when compared with CRP and WBC.

Another biomarker we observed in the study was IL-6. IL-6 is a cytokine that can be produced by macrophages, lymphocytes, endothelial cells and other tissues. It is involved in the modulation of the acute phase response and can be detected in the serum of patients with burn injury and sepsis, during the postoperative period. Previous studies showed that serum levels of IL-6 rose earlier than CRP and PCT after bacterial infection and decreased more quickly. Further research show that IL-6 not only has good early diagnostic value in patients with infection and sepsis [Steinmetz 1995], but also can help clinicians [Steinmetz 1995; Le Moine 1994] to differentiate infection from patients with SIRS [Ma 2016]. These are in accordance with the results in Table 2 and Figure 2, which indicate that IL-6 was a better predictor of infections when measured at POD3 to POD5 and when compared with CRP and WBC, but it was not as good as the PCT.

Cardiac surgery with CPB leads to inflammatory cytokine release and stimulates SIRS [Wan 1997]; resulting diagnosis of PP is challenging because conventional clinical and biological signs may be misleading by SIRS [Li 2017]. In our study, the diagnostic value of PCT was higher than WBC and CRP with diagnosing PP, and PCT variations were better than PCT absolute value (Table 3). The superiority of PCT may be explained by its specific increase after bacterial infection, which also leads PCT variations changed gentler in the PP group. Lack of the specificity may be the main reason why
IL-6 didn't show the similar diagnosis ability with PCT (Table 3). Although IL-6 seems not as good as PCT, IL-6 variation from POD 3 to POD1 and IL-6 variation from POD4 to POD1 show excellent specificity [95\%; 96\%]. This characteristic may help us exclude false positive patients in the PP group. Nevertheless, it should be pointed out that the diagnostic ability of PCT variations during the first 3 PODs are not satisfying because the maximal AUC of PCT and PCT variations were taken on POD4 and POD5, respectively.

Haghi et al reported that combining PCT and IL-6 improved the diagnostic efficiency of infectious appendicitis and decreased unnecessary negative appendectomies [Haghi 2018]. Another study demonstrated that the combination of IL-6, CRP and PCT seems to be predictive in diagnosis of early onset neonatal sepsis [Abdollahi 2012]. These studies remind us that the combination of PCT and IL-6 may help improve diagnostic efficacy. Then, we used multivariable logistic regression analysis to find risk factors independently correlated with PP. Multivariable logistic regression found PCT variation from POD3 to POD1, IL-6 variation from POD3 to POD1, previous cardiac surgery, MV time and cardiopulmonary bypass time were independently correlated with PP. Among them, PCT variation and IL-6 variation were the strongest risk factors for PP [OR: $12.50,95 \% \mathrm{CI}$ : (3.40-45.5); OR: 13.71, 95\% CI: (1.11-168.47)] (Table 4).

In order to predict PP earlier and more accurately, we defined the PL Index based on the following formula, according to the above results: PL Index PODn $=13 *$ PCT Variation PODn to POD1+14*IL-6 Variation PODn to POD1. We calculated different PL Index indices, according to the above formula (result shown in Table 3), PL Index POD3 had the maximal AUC [ROC: 0.90, 95\% CI: (0.79-0.95)] (Table 3 and Figure 5), the sensitivity and specificity of PL Index POD3 were $88 \%$ and $68 \%$. Then, we used multivariable logistic regression analysis to test the correlation between PL Index POD3 and PP. Results of the multivariable logistic regression showed that PL Index POD3 had higher correlation compared with PCT variation from POD3 to POD1 [OR:1.23, 95\% CI: $(1.11-1.37), P=.041$ versus OR: $1.32,95 \%$ CI: (0.15-11.34), $P=.799]$. In summary, PL Index showed the optimal diagnostic value with $\mathrm{PP}$ in patients who underwent cardiac surgery with CPB in the POD3.

Our study provided some interesting information, regarding the combination of PCT and IL-6, that is important for improving early diagnosis of PP. First, it is obvious that, because of the inflammatory process related to $\mathrm{CPB}$, all biomarkers increased during the first 2 PODs, thus weakening the diagnostic abilities of these biomarkers (Table 2 and Table 3 ). In the POD3, PL Index could be used as an indicator of postoperative infection, which was more accurate than using any biomarker alone and earlier than using PCT variation (Table 3 and Figure 5). From our study, we thought the diagnostic value of the PL Index was helpful with guiding which patients should adjust the antibiotics, as PCT has shown in patients with lower respiratory tract infections already [Christ-Crain 2004]. Meanwhile, we noticed that the diagnosis of infected patients could occur earlier with continuous PCT and IL-6 monitoring, allowing an earlier administration of antibiotics 
in infected patients, and it should be pointed out that taking further therapeutic measures earlier could improve the prognosis in PP and sepsis [Rivers 2001].

Several limitations should be considered to interpret this study. First, the present study was a single-center based observational analysis, and the results support an association and not necessarily causation. These results need to be validated in larger, multicenter studies. Second, there were many manipulations of the PCT and IL-6 data that decreased their clinical utilities. Third, because most of the PCT and IL-6 measurements were performed within 5 days after surgery, the correlation of PCT and IL-6 to the prognosis of PP deserves further study.

\section{CONCLUSIONS}

PCT and IL-6 were more accurate than CRP and WBC with predicting early $\mathrm{PP}$, but the diagnostic properties of PCT could not be observed during the first 3 PODs due to the inflammatory process related to $\mathrm{CPB}$. The dynamic change of PCT is more important than the absolute value to predict postoperative infection. Diagnostic value of IL-6 and IL-6 variations was not satisfying. Through the combination of PCT and IL-6 variations, PL Index was the best predictor of PP at POD3.

\section{REFERENCES}

Abdollahi A, Shoar S, Nayyeri F, Shariat M. 2012. Diagnostic Value of Simultaneous Measurement of Procalcitonin, Interleukin-6 and hs-CRP in Prediction of Early-Onset Neonatal Sepsis. MEDITERR J HEMATOL I. 4(1):e2012028.

Ailawadi G, Chang HL, O'Gara PT, O'Sullivan K, Woo YJ, DeRose JJJ, et al. 2017. Pneumonia after cardiac surgery: Experience of the National Institutes of Health/Canadian Institutes of Health Research Cardiothoracic Surgical Trials Network. The Journal of thoracic and cardiovascular surgery. 153(6):1384-91.

Assicot M, Gendrel D, Carsin H, Raymond J, Guilbaud J, Bohuon C. 1993. High serum procalcitonin concentrations in patients with sepsis and infection. Lancet (London, England). 341(8844):515-8.

Baykut D, Schulte-Herbrüggen J, Krian A. 2000. The value of procalcitonin as an infection marker in cardiac surgery. EUR J MED RES. 5(12):530-6.

Becker KL, Snider R, Nylen ES. 2008. Procalcitonin assay in systemic inflammation, infection, and sepsis: clinical utility and limitations. CRIT CARE MED. 36(3):941-52.

Calandra T, Cohen J. 2005. The international sepsis forum consensus conference on definitions of infection in the intensive care unit. p. 1538-48.

Chanderraj R, Dickson RP. 2018. Rethinking pneumonia: A paradigm shift with practical utility. Proc Natl Acad Sci U S A. [Journal Article; Research Support, N.I.H., Extramural; Comment]. 12-26;115(52):13148-50.

Christ-Crain M, Jaccard-Stolz D, Bingisser R, Gencay MM, Huber PR, Tamm M, et al. 2004. Effect of procalcitonin-guided treatment on antibiotic use and outcome in lower respiratory tract infections: cluster-randomised, single-blinded intervention trial. p. 600-7.

Dandona P, Nix D, Wilson MF, Aljada A, Love J, Assicot M, et al. 1994. Procalcitonin increase after endotoxin injection in normal subjects. The Journal of clinical endocrinology and metabolism. 79(6):1605-8.

Dörge H, Schöndube FA, Dörge P, Seipelt R, Voss M, Messmer BJ. 2003. Procalcitonin is a valuable prognostic marker in cardiac surgery but not specific for infection. The Thoracic and cardiovascular surgeon. 51(6):322-6.

Edwards FH, Engelman RM, Houck P, Shahian DM, Bridges CR. 2006. The Society of Thoracic Surgeons Practice Guideline Series: Antibiotic Prophylaxis in Cardiac Surgery, Part I: Duration. The Annals of thoracic surgery. 81(1):397-404.

Engelman R, Shahian D, Shemin R, Guy TS, Bratzler D, Edwards F, et al. 2007. The Society of Thoracic Surgeons practice guideline series: Antibiotic prophylaxis in cardiac surgery, part II: Antibiotic choice. The Annals of thoracic surgery. 83(4):1569-76.

Guidelines for the management of adults with hospital-acquired, ventilator-associated, and healthcare-associated pneumonia. 2005. p. 388-416.

Haghi AR, Kasraianfard A, Monsef A, Kazemi AS, Rahimi S, Javadi SMR. 2018. The diagnostic values of procalcitonin and interleukin 6 in acute appendicitis. Turkish journal of surgery. 35(1):1-3.

He S, Chen B, Li W, Yan J, Chen L, Wang X, et al. 2014. Ventilatorassociated pneumonia after cardiac surgery: a meta-analysis and systematic review. The Journal of thoracic and cardiovascular surgery. 148(6):3148-55.

Hulzebos EHJ, Helders PJM, Favié NJ, De Bie RA, Brutel de la Riviere A, Van Meeteren NLU. 2006. Preoperative intensive inspiratory muscle training to prevent postoperative pulmonary complications in high-risk patients undergoing CABG surgery: a randomized clinical trial. JAMA. 296(15):1851-7.

Jebali MA, Hausfater P, Abbes Z, Aouni Z, Riou B, Ferjani M. 2007. Assessment of the accuracy of procalcitonin to diagnose postoperative infection after cardiac surgery. p. 232-8.

Kalil AC, Metersky ML, Klompas M, Muscedere J, Sweeney DA, Palmer LB, et al. 2016. Management of Adults With Hospital-acquired and Ventilator-associated Pneumonia: 2016 Clinical Practice Guidelines by the Infectious Diseases Society of America and the American Thoracic Society. Clinical infectious diseases : an official publication of the Infectious Diseases Society of America. 63(5):e61-111.

Kin H, Kawazoe K, Nakajima T, Niinuma H, Kataoka T, Endo S, et al. 2003. Perioperative serum procalcitonin concentrations in patients with acute aortic dissection. EUR SURG RES. [Journal Article]. 35(5):451-4.

Kinlin LM, Kirchner C, Zhang H, Daley J, Fisman DN. 2010. Derivation and validation of a clinical prediction rule for nosocomial pneumonia after coronary artery bypass graft surgery. Clinical infectious diseases: an official publication of the Infectious Diseases Society of America. 50(4):493-501.

Klingele M, Bomberg H, Schuster S, Schäfers H, Groesdonk HV. 2016. Prognostic value of procalcitonin in patients after elective cardiac surgery: a prospective cohort study. ANN INTENSIVE CARE. 6(1):116.

Le Moine O, Deviere J, Devaster JM, Crusiaux A, Durand F, Bernuau J, et al. 1994. Interleukin-6: an early marker of bacterial infection in decompensated cirrhosis. J HEPATOL. [Clinical Trial; Controlled Clinical Trial; Journal Article; Research Support, Non-U.S. Gov't]. 06-01;20(6):819-24.

Li X, Wang X, Li S, Yan J, Li D. 2017. Diagnostic Value of Procalcitonin 
on Early Postoperative Infection After Pediatric Cardiac Surgery. p. 420-8.

Ma L, Zhang H, Yin Y, Guo W, Ma Y, Wang Y, et al. 2016. Role of interleukin-6 to differentiate sepsis from non-infectious systemic inflammatory response syndrome. CYTOKINE. 88:126-35.

Macrina F, Tritapepe L, Pompei F, Sciangula A, Evangelista E, Toscano F, et al. 2005. Procalcitonin is useful whereas C-reactive protein is not, to predict complications following coronary artery bypass surgery. Perfusion. 20(3):169-75.

Rivers E, Nguyen B, Havstad S, Ressler J, Muzzin A, Knoblich B, et al. 2001. Early goal-directed therapy in the treatment of severe sepsis and septic shock. p. 1368-77.

Steinmetz HT, Herbertz A, Bertram M, Diehl V. 1995. Increase in interleukin-6 serum level preceding fever in granulocytopenia and correlation with death from sepsis. The Journal of infectious diseases. 171(1):225-8.

Tanaka T, Narazaki M, Kishimoto T. 2014. IL-6 in inflammation, immunity, and disease. CSH PERSPECT BIOL. 6(10):a16295.

van Rossum AMC, Wulkan RW, Oudesluys-Murphy AM. 2004. Procalcitonin as an early marker of infection in neonates and children. The Lancet. Infectious diseases. 4(10):620-30.

Vos RJ, Van Putte BP, Kloppenburg GTL. 2018. Prevention of deep sternal wound infection in cardiac surgery: a literature review. The Journal of hospital infection. 100(4):411-20.

Wacker C, Prkno A, Brunkhorst FM, Schlattmann P. 2013. Procalcitonin as a diagnostic marker for sepsis: a systematic review and meta-analysis. The Lancet. Infectious diseases. 13(5):426-35.

Wan S, LeClerc JL, Vincent JL. 1997. Inflammatory response to cardiopulmonary bypass: mechanisms involved and possible therapeutic strategies. CHEST. 112(3):676-92.

Youden WJ. 1950. Index for rating diagnostic tests. CANCER-AM CANCER SOC. 3(1):32-5. 